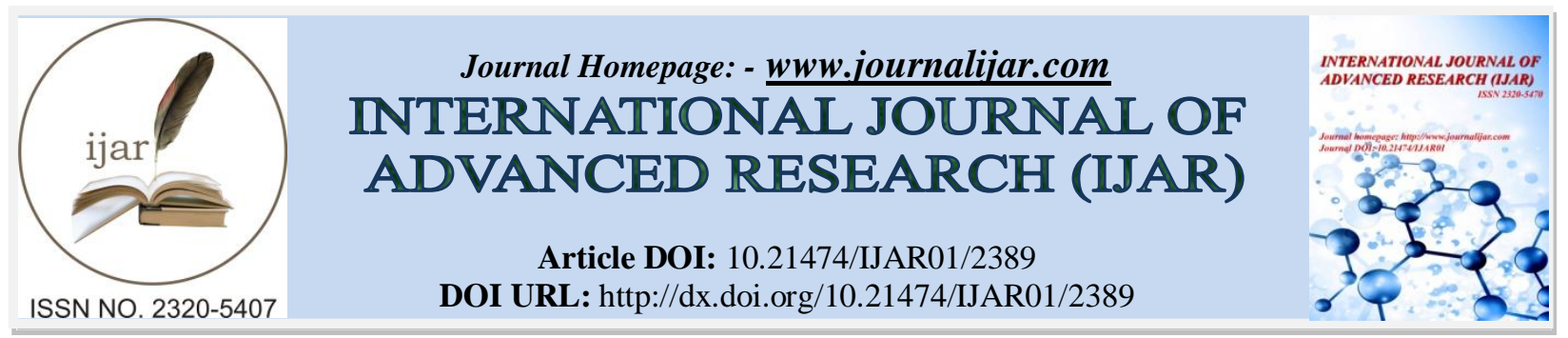

RESEARCH ARTICLE

\title{
THE EFFECT OF HIGH DOSE OF GREEN TEA ON THERAPEUTIC ACTION OF GAMMA RADIATION IN LIVER CARCINOMA.
}

\author{
Rana Essam ${ }^{2}$, Fathia EL Sharkawi ${ }^{1}$, Mahmoud Shabon ${ }^{2}$ and Hussien Khaled ${ }^{3}$. \\ 1. Department of Biochemistry and Molecular Biology, Faculty of Pharmacy, Helwan University, Egypt. \\ 2. Egyptian Nuclear and Radiological Regulatory Authority. \\ 3. Medical Oncology Department, National Cancer Institute, Cairo University, Egypt.
}

\section{Manuscript Info}

\section{Manuscript History}

Received: 19 October 2016

Final Accepted: 20 November 2016

Published: December 2016

\section{Key words:-}

Hepatocellular carcinoma, Tumor generating factor- $\beta$, Green tea extract, Gamma radiation.

\section{Abstract}

Hepatocellular carcinoma (HCC) is one of the most common malignancies worldwide and the second leading cause of death from cancer. Radiotherapy is considered as a corner stone of cancer treatment, one of the future challenges is to minimize the harmful effects of radiotherapy by reducing its dose and combined use of natural antitumor agent. Plant extracts eliciting radioprotective efficacy which can be used to overcome those harmful effects. in this study green tea extract was used in combination with radiotherapy in HCC induced rats model. forty eight female albino HCC induced rats were used, They divided into four groups: control HCC group, group exposed to $10 \mathrm{~Gy}$ gamma radiation, group ingested with high dose of green tea extract $(2.06 \mathrm{~g} / \mathrm{Kg})$ and the forth group received a combined treatment of green tea extract and gamma radiation. Serum alphafetoprotein (AFP) was measured by ELIZA technique and tumor generating factor- $\beta$ (TGF $\beta$ ) expression was determined using RTPCR method.

Results: AFP and TGF- $\beta$ were decreased in HCC treated group with radiation while the group treated with green tea extract showed decline in serum AFP level and increase in TGF- $\beta$ expression level. In HCC treated group with combination of radiation and green tea extract, there was a decrease in AFP level with extreme elevation in TGF- $\beta$.

In conclusion, the use of high dose of green tea extract in combination with radiation in HCC might prevent the antitumor therapeutic effect of radiation and produced an extremely elevation in the TGF- $\beta$ expression.

Copy Right, IJAR, 2016,. All rights reserved.

\section{Introduction:-}

Cancer is a group of diseases involving abnormal cell growth with the potential to invade or spread to other parts of the body. The most common types of cancer in males are lung, liver, prostate, colorectal and stomach and in females, breast, colorectal, stomach and liver cancers worldwide (WHO, 2014). 
Hepatocellular carcinoma (HCC) is one of the most common malignancies worldwide and the second leading cause of death from cancer (Satoshi et al., 2016).

Among the major genes that show remarkable expression during HCC stages is transforming growth factor-beta "TGF- $\beta$ " which is a cytokine responsible for homeostasis preservation, inducing cell cycle arrest and apoptosis in different organs, including the liver. It`s considered a tumor suppressor. TGF- $\beta$ also induces anti-apoptotic signals in rat hepatoma cells (Pardali et al., 2007).

There are multiple therapeutic modalities available for $\mathrm{HCC}$, and the selection of a particular therapy depends on the stage of HCC.

Radiotherapy (RT) is considered as a corner stone of cancer treatment, and more than $50 \%$ of cancer patients use RT at least once during their treatment. RT can be applied alone in a curative intent or associated with chemotherapy and/or surgery performed before or after RT. (Feys et al., 2015)

Recent findings emphasize the interest to optimize RT ( dose per fraction and timing of surgery). The future challenges in RT will define the most appropriate combinations between RT and other therapeutic modalities with the optimal sequence and timing of treatments (Surace et al., 2015).

One of these challenges is to minimize the harmful effects of RT which can be obtained by combination with one of radio protector agents.

Plant extracts eliciting radioprotective efficacy contain a variety of compounds including antioxidants, antiinflammatory, immunostimulants, cell proliferation stimulators and antimicrobial agents (Hafezeh et al., 2012).

Green tea exerts a protective effect in the liver, it protects against liver damage caused by alcohol and carbon tetrachloride in rats (Xiao et al., 2002). The protective effect of drinking green tea was related to its catechins.Catechins in green tea act effectively as a free radical scavenger to reduce the damages caused by ionizing-induced free radicals. On the other hand green tea polyphenols showed to be able to reduce the harmful effect of whole body irradiation in rats. From all green tea polyphenol the catechins revealed to be most effective (Hafezeh et al., 2012).

Regarding all the evidences about the antioxidant and radioprotective effects of green tea, the question remained unanswered is that whether drinking green tea is able to improve HCC cases when treated with ionizing radiation?

So, in this study we aimed to use a combination of gamma radiation and green tea extract in definite doses to study their potential therapeutic effects in HCC induced rats.

\section{Materials and Methods:-}

\section{Chemicals:-}

1. Green tea extract: obtained from Isis Company, Egypt.

2. Diethyl nitrosamine: obtained from Sigma-Aldrich, Cairo, Egypt

3. CCL4 provided from Nuclear and radiological regulatory authority.

4. Chloroform and methanol from EL-Gomhoreya co. Egypt.

Reagents for biochemical analysis:-

1. Rat Alpha-fetoprotein (AFP) ELISA Kit, Catalog No. CSB-E08281r (96T) CUSABIO BIOTECH CO., LTD.

2. Direct-zol RNA miniprep for RNA extraction from tissue. Purchased from Sigma analysis co. Egypt

3. SensiFAST cDNA synthesis from Sigma analysis co. Egypt

4. Sensifast SYBR green norox from Sigma analysis co. Egypt.

\section{Experimental Animals:-}

Eighty four female albino rats of matched age and weight (1 to 1.5 months old) and (130-150 g) obtained from the National Research Center (Giza, Egypt) were included in the study. Animals were inbred in the animal house of 
Egyptian Atomic Energy Authority according to the standard guidelines of Institutional Animal Care under controlled conditions of humidity, temperature and a diurnal environment of light and dark.

Induction of HCC:-

Hepatocellular carcinoma was induced for forty eight rats according to the methods of Sundaresan et al., 2003 and Dakshayani et al., 2005.

Irradiation of animals:-

Rats were exposed to whole body gamma radiation in cesium-137 irradiation unit in Egyptian Atomic Energy Authority.

Group classification of the experimental animals:-

Thirty six healthy rats were used as control and subdivided into the following groups:

Healthy control group (C): Twelve healthy rats.

Irradiated control group (CR): 12 rats exposed to Gamma radiation 1 Gy two times / week for five consecutive weeks.

Green tea control group (G): 12 rats received green tea extract orally $(2.06 \mathrm{~g} / \mathrm{Kg})$ for five consecutive weeks.(Vanessa et al., 2004).

The $48 \mathrm{HCC}$ induced rats were subdivided equally into four subgroups twelve rats in each group:

Hepatocellular carcinoma group (HCC): Induction of HCC only and not treated.

Gamma Radiation treated group (R): Exposed to gamma radiation $1 \mathrm{~Gy}$ two times/ week for 5 weeks.

Green tea treated group $(\mathrm{G})$ : Received green tea extract orally $(0.1 \% \mathrm{wt}: \mathrm{v})$ for five consecutive weeks.

Radiation and green tea group (RG): Received both radiation and green tea extract for 5 weeks..

\section{Sampling:-}

At the end of the experiment, animals were sacrificed by decapitation. Blood samples were collected and centrifuged at $5000 \mathrm{rpm}$ for 10 minutes to separate serum for further analysis.

Liver tissue samples were collected for both QRT-PCR and histopathological study.

\section{Biochemical analysis:-}

Determination of serum AFP by ELISA method:-

AFP was determined in serum by enzyme-linked immunoassay technique. (Takahashi et al., 1988)

Quantitative real-time PCR based analysis:-

RT-qPCR assay was carried out for relative quantification of TGF- $\beta$ expression using its specific primers, Glyceraldehyde 3-phosphate dehydrogenase (GAPDH) was used as housekeeping gene.

\section{Calculation of Results:-}

Changes in the target mRNA content relative to GAPDH gene were determined based on the cycle threshold $(\mathrm{Ct})$ level according to (livak and schmittgen 2001).

\section{Statistical analysis:-}

Data were collected for all analyzed parameters and tabulated.

Appropriate graphs were plotted when needed using Graphpad prism 6 Prism 6 for windows (version 6.01; Graphpad software, inc., La Jolla. CA, USA).

Data were expressed as mean \pm standard error, to analyse two sets of data, unpaired Students t- test was used. While ordinary one way ANOVA was used to analyze more than two sets of data, for parametric data followed by TukeyKramar multiple comparison test.

Descriptive Statistics:-

Mean (Mn)

Standard error (SE)

$* * *$ Very highly significant $\mathrm{P}<0.001$ 
** Highly significant $\mathrm{P}<0.01$

* significant $\mathrm{P}<0.05$

non significant $\mathrm{P}>0.05$

\section{Results:-}

Effect of gamma radiation and green tea extract on serum alpha feto protein (AFP) levels in all experimental groups:-

Levels of serum AFP are presented in table (1) and illustrated in figure (1)

The data showed that in control group (C), green tea group (CG) and irradiated group (CR), AFP serum levels were $1.07 \pm 0.07,0.90 \pm 0.03$ and $1.10 \pm 0.03 \mathrm{\eta g} / \mathrm{ml}$ respectively. Very highly significant increase in AFP serum levels was shown in the hepatocellular carcinoma (HCC) group $57.03 \pm 3.85 \mathrm{\eta g} / \mathrm{ml}$.

In HCC treated group with radiation (R) there was a very highly significant decrease in AFP serum level $34.85 \pm 2.02 \eta \mathrm{g} / \mathrm{ml}$ compared with $\mathrm{HCC}$ group $57.03 \pm 3.85 \eta \mathrm{g} / \mathrm{ml}$. Also in $\mathrm{HCC}$ treated group with green tea extract $(\mathrm{G})$, a very highly significant decrease in serum AFP levels was shown $(16.48 \pm 0.86 \eta \mathrm{g} / \mathrm{ml})$ than HCC group ( $57.03 \pm 3.85 \eta \mathrm{g} / \mathrm{ml})$.

On the other hand it was observed that the use of combination of radiation and green tea extract (RG) caused a very highly significant decrease in AFP serum level $9.20 \pm 0.43 \eta \mathrm{g} / \mathrm{ml}$ than the use of radiation or green tea extract alone without combination.

Table 1:- Serum AFP level in all experimental animal groups.

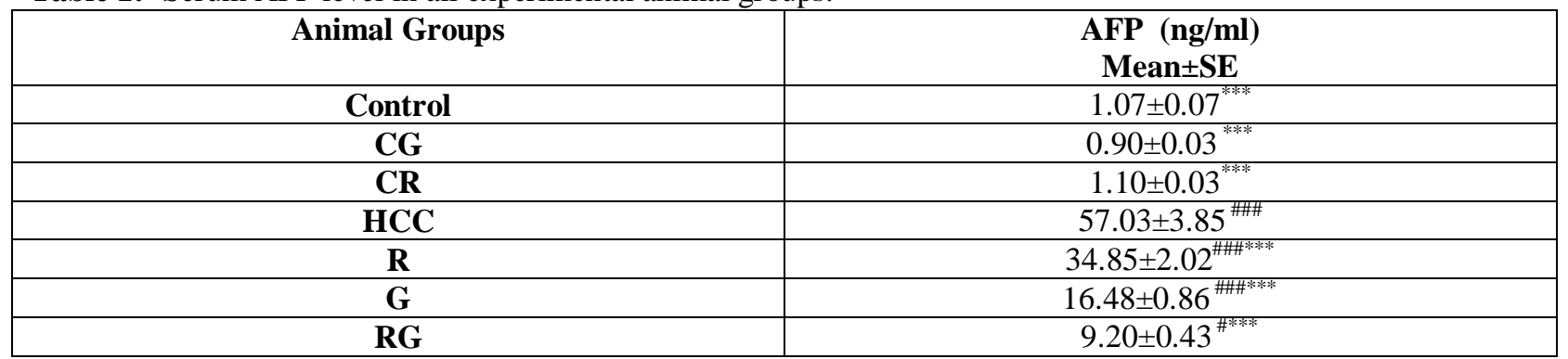

- Results were presented as mean \pm SE.

- \# Values differ significantly from healthy control

- *Values differ significantly from HCC

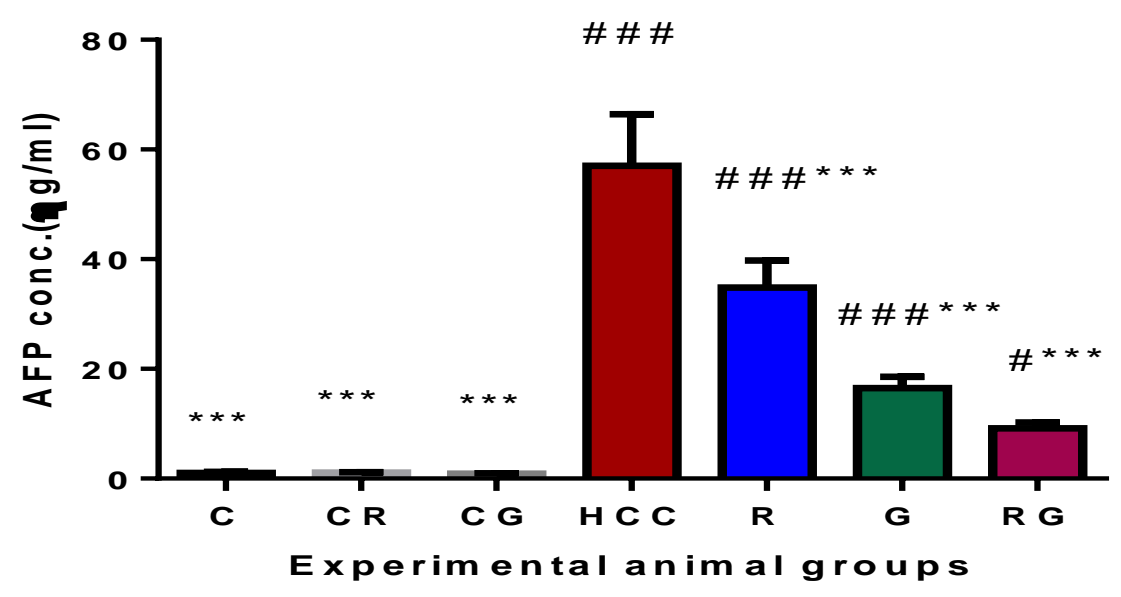

C: Control group

Figure 1:- Serum AFP levels (ng/ml) in different studied groups. 
CG: normal rats with Green Tea

CR: normal rats with Gamma radiation

HCC: Hepatocellular carcinoma rats (diseased and untreated group)

$\mathrm{R}$ : HCC with Gamma radiation

G: HCC with Green tea

RG: HCC with Radiation and Green tea

QRT-PCR gene expression of TGF- $\beta$ gene:-

The effect of gamma radiation and green tea extract on HCC treatment was assessed by measuring TGF- $\beta$ expression levels.

\section{TGF- $\beta$ relative expression:-}

The data are presented in table (2) and illustrated in figure (2):

1. In HCC group there was a highly significant increase in the expression of TGF- $\beta$ in liver homogenate $2.52 \pm 0.64$ compared with control group $0.89 \pm 0.12$, while $\mathrm{R}$ group showed significant decrease in of TGF- $\beta$ expression $0.52 \pm 0.17$ compared to HCC group.

2. Treatment of the HCC animal groups with green tea extract $(\mathrm{G})$ showed an a very high increase in TGF- $\beta$ expression level 39.67 10.68 while when a combination of radiation and green tea extract was used there was a very high over expression in TGF- $\beta 234.1 \pm 68.11$ than that of green tea group.

Table 2:- Effect of treatment with radiation $(\mathrm{R})$ and green tea $(\mathrm{G})$ on TGF- $\beta$ expression levels:

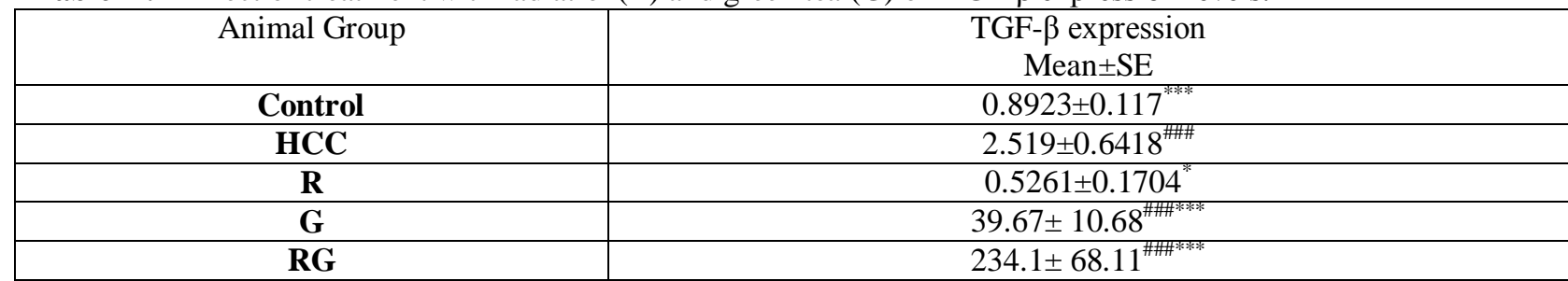

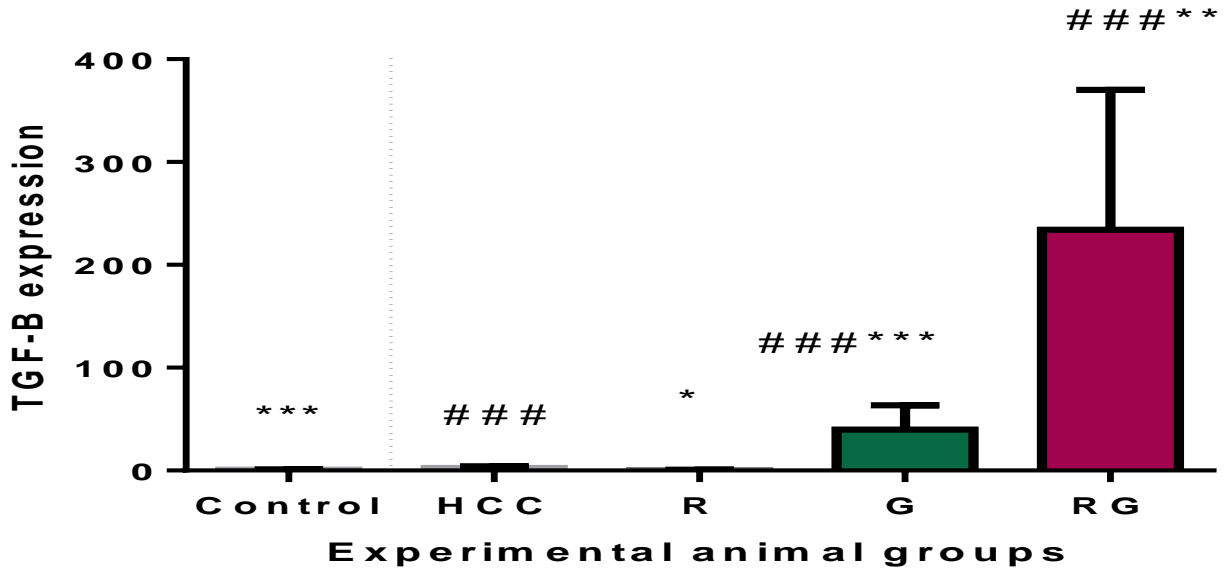

Figure 2:- The TGF- $\beta$ expression levels in studied groups.

\# Values differ significantly from healthy control

* Values differ significantly from HCC.

C: Control group

HCC: Hepatocellular carcinoma rats (diseased and untreated group)

$\mathrm{R}$ : HCC with gamma radiation

G: HCC with green tea extract

$\mathrm{RG}$ : $\mathrm{HCC}$ with radiation and green tea. 


\section{Histopathological examination:-}

Histopathological examination of liver tissues of: $\mathrm{C}$ group showing normal histological structure of the central vein and surrounding hepatocytes in the hepatic parenchyma (Figure 3).

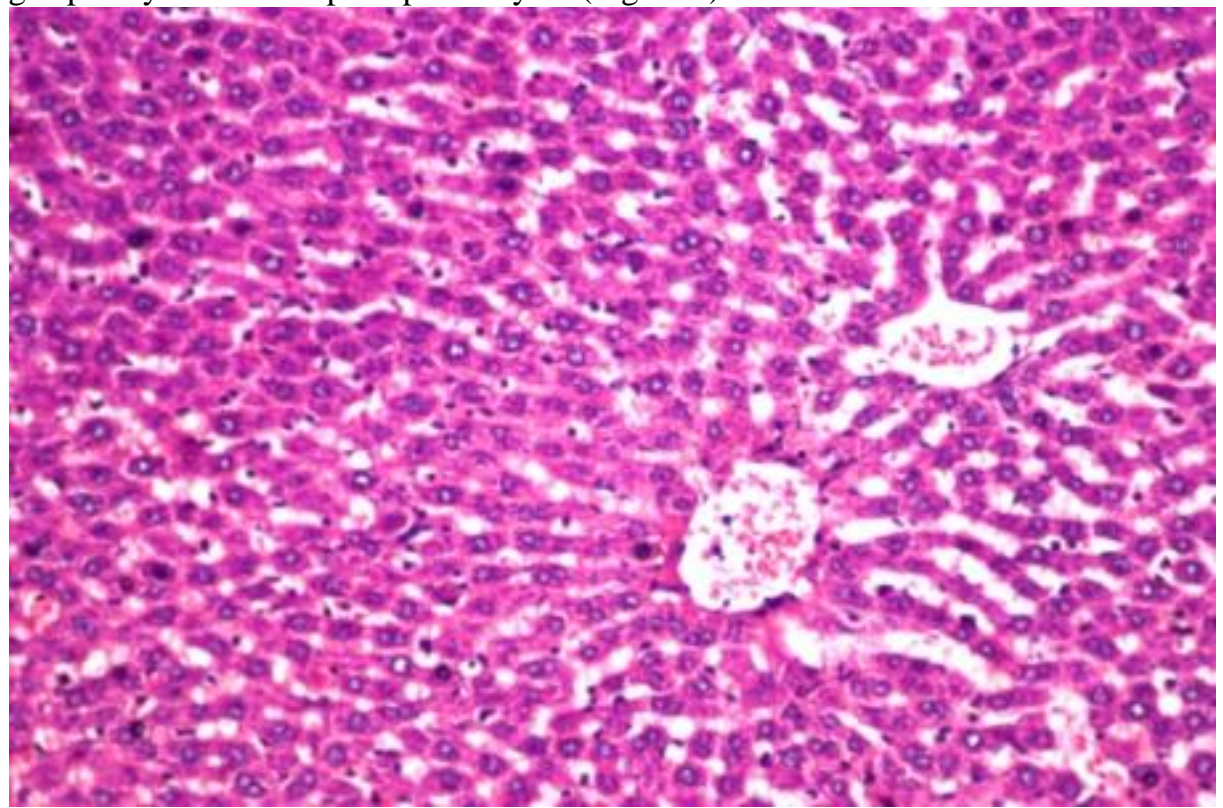

Figure 3:-

Histopathology of liver tissues of the animals that received only DEN and $\mathrm{CCl}_{4}$ showed $\mathrm{HCC}$ with severe multiple nodular foci of anaplastic hepatocytes defined by fibrous tissue with inflammatory cells infiltration in the portal area were recorded. the hepatocytes in some nodules showed karyomegalocytes, degenerative change and dysplasia(Figure 4).

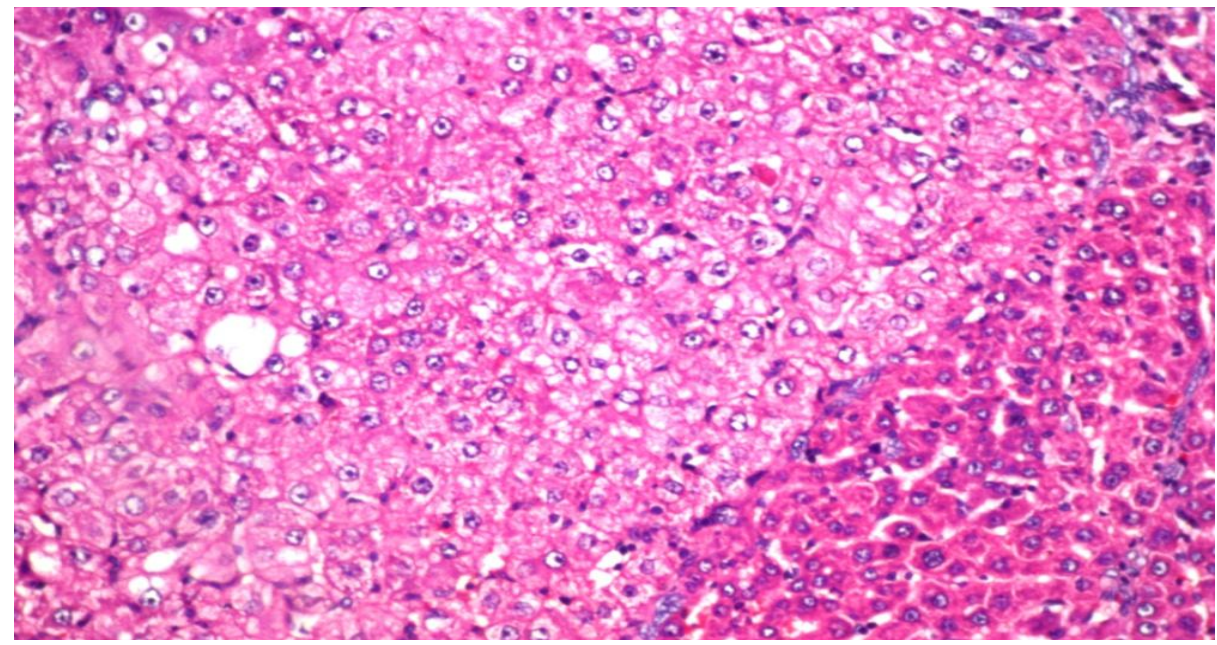

Figure 4:-

Liver tissues of RG group (figure 5) showed mild adenoma with less nodular foci of anaplastic hepatocytes and absence of fibrosis. 


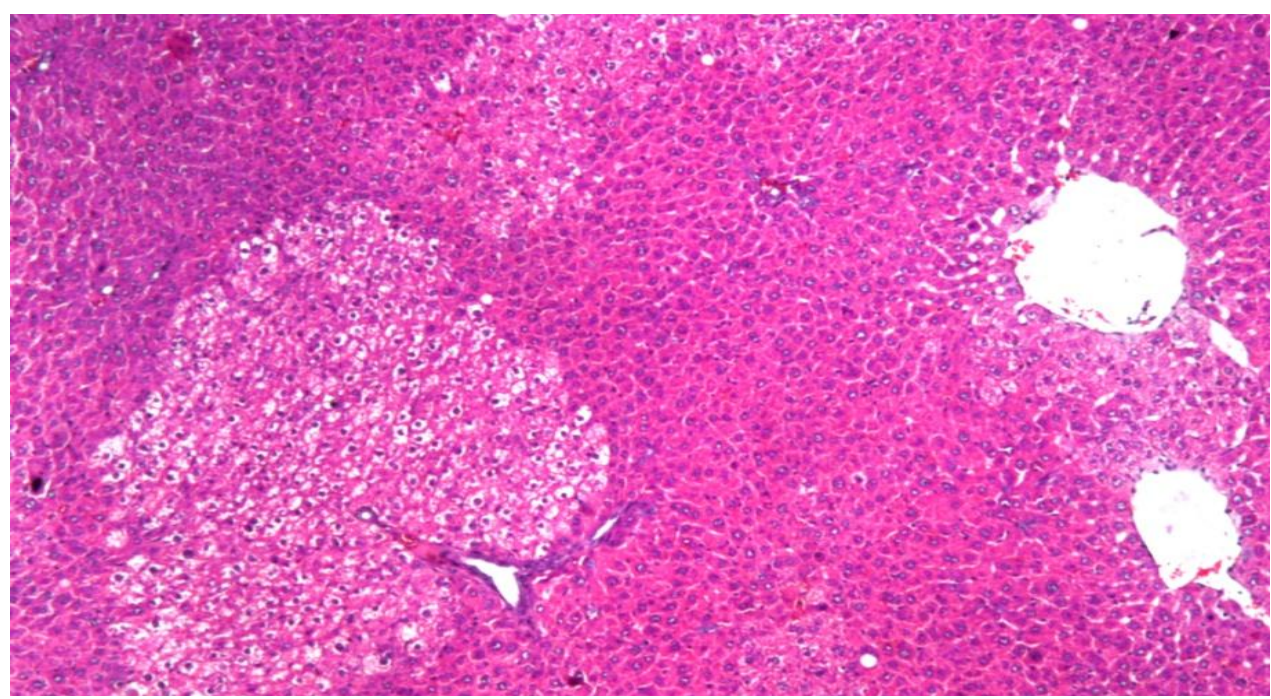

Figure 5:-

\section{Discussion:-}

Traditionally, the role of radiotherapy (RT) in liver tumors has been limited by the risk of radiation-induced liver toxicity and the low tolerance of the whole liver to RT. In recent years, improvements in diagnostic imaging, RTplanning techniques (which allow delivery of low doses of gamma radiation) targeting the tumor and minimizing dose to surrounding tissues and organs (Feys et al., 2015). Fractionated dose of radiation is applied in the present study in addition to green tea extract to overcome the adverse effects of ionizing radiation and to minimize its harmful effects on the normal cells. Green tea extract is a potent antioxidant, radio protector and an anticancer agent (Feuerer et al., 2009). EGCG_(Epigalocatechin gallate, which is the main active constituent of green tea and represents 70 to $80 \%$ of its all constituents. (Vanessa et al., 2004)) can alter gene expression by inhibiting DNMT (DNA methylation) activities, resulting in the reactivation of methylation-silenced genes via epigenetic modifications (Berletch et al.,2008). EGCG has been associated with a variety of health promoting activities, including anti-inflammatory and anti-carcinogenic effects. Several studies demonstrated that EGCG and green tea polyphenols have an in vitro immune suppressive effects on T cells, also in vivo studies showed that EGCG had an inhibitory effects on autoimmune disease progression (Pae et al., 2010). Furthermore, in addition to DNMT inhibitory activities, EGCG is known to exert multiple effects on numerous additional targets, including the induction of cell cycle arrest and apoptosis, and inhibition of MAP kinase and growth factor-related signaling (Fu et al., 2010).

Regulatory T cells play a pivotal role in the maintenance of immunetolerance and the suppression of autoimmunity (Feuerer et al., 2009). Disruption of T-regulatory development and function results in immune dysregulation and autoimmune diseases. T-regulatory differentiation is regulated by Foxp3, a member of the forkhead /winged-helix family of transcription factors which serves as a master regulator for T-regulatory generation. Many reports demonstrated that the expression and stability of Foxp3 is epigenetically regulated (Lal et al., 2009). which can be potentially exploited in generating suppressive T-regulatory.

Foxp3 is a specific marker of T-regulatory cells and many studies confirm that Foxp3 induction is associated with immunosuppressive activity of T-regulatory cells. The DNMT inhibitory effect of EGCG on Foxp3 expression represents a novel and previously uncharacterized mechanism of action that can potentially contribute to the induction and/or enhancement of T-regulatory frequency and function (Carmen et al., 2011).

Foxp3 is neither the only target of EGCG, nor that the increase in T-regulatory is solely due to epigenetic mechanisms, as EGCG likely act via a combination of mechanisms in modulating immune response (Carmen et al., 2011).

On the other hand T-regulatory cells secrete transforming growth factor TGF- $\beta$ and interleukin 10 . It was proposed that tumor-specific T-regulatory cells accumulate locally in HCC and inhibit the protective specific responses (Nakamura et al., 2004). 
The immune-modulating effect of EGCG proposed by the early studies was largely based on the altered lymphocyte proliferation after in vitro supplementation with tea extracts or catechins. Hu and colleagues showed that EGCG at dose of $24 \mu \mathrm{g} / \mathrm{ml}$ and higher inhibited both B and T cell proliferation, but a greater effect was observed in T cells (Hu et al., 1992). Also several results showed that administration of $0.3 \%$ EGCG for 6 weeks was effective in reducing T cell proliferation (Pae et al., 2010). But the contrast was observed with higher dose of EGCG. Carmen et al.,2011 reported in his in vivo study that the EGCG in a dose of $50 \mathrm{mg} / \mathrm{kg}(1 \mathrm{mg} / \mathrm{mouse})$ for 7 days resulted in DNMT inhibition and induction of both Foxp3 and IL-10 expression which lead to an increase in t-regulatory cells numbers (Carmen et al., 2011). The current study showed that the use of high dose of EGCG $(1.65 \mathrm{~g} / \mathrm{kg})$ for long duration (5weeks) caused increase in TGF- $\beta$ expression and antagonizes the effect of radiation on HCC cells.

A TGF- $\beta$ rich tumor environment would be ideal for the maintenance of induction of T-regulatory cells. Giannelli et al., 2002 reported that TGF- $\beta$ stimulates 3 -integrin expression at a transcriptional level in non invasive HCC, causing transformation into an invasive phenotype through the secretion of TGF- $\beta$ by T-regulatory cells which may potentiate HCC invasiveness.3-integrin might promote tumorigenesis through a direct effect on the growth of carcinoma cells and a TGF- $\beta$-dependent effect on the invasion of carcinoma cells The integrins carry out essential roles during both normal and neoplastic cell migration. As well as providing the anchorage that is necessary for migration, they activate pro-migratory signals that regulate the cytoskeleton as well as gene expression. The mechanisms by which integrin signals orchestrate cell migration are still incompletely understood (Wenjun et al., 2004).

TGF- $\beta$ signaling in carcinogenesis is complex; in early-stage HCC, It acts as a tumor suppressor, but in the late phase it plays a role as a tumor promoter (M.Sakamoto et al., 2010).

\section{Conclusion:-}

Depending on all mentioned mechanisms the authors of the present study concluded that the use of high dose of green tea extract in combination with radiation in HCC might prevent the antitumor therapeutic effect of radiation and produced an extremely elevation in the TGF- $\beta$ expression as a result of T-regulatory cells promotion .

\section{References:-}

1. Berletch JB, Liu C, Love WK, Andrews LG, Katiyar SK, Tollefsbol TO. (2008) Epigenetic and genetic mechanisms contribute to telomerase inhibition by EGCG. J Cell Biochem; 103:509-19.

2. Carmen P. Wonga, Linda P. Nguyena et al, (2011) Induction of regulatory T cells by green tea polyphenol EGCG. Immunology Letters 139 7- 13.

3. Dakshayani KB, Subramanian P, Manivasagam T, Essa MM, Manoharan S. (2005) Melatonin modulates the oxidant-antioxidant imbalance during Nitrosodiethylamine induced hepatocarcinogenesis in rats. $J$ Pharm Pharm Sci, 8(2):316- 21.

4. Dayong Wua, Junpeng Wanga, Munkyong Pae a,1, Simin Nikbin Meydani. (2012) Green tea EGCG, T cells, and T cell-mediated autoimmune diseases. Molecular Aspects of Medicine 33 107-118.

5. Feuerer M, Hill JA, Mathis D, Benoist C.( 2009) Foxp3+ regulatory T cells: differentiation, specification, subphenotypes. Nat Immunol;10:689-95.

6. Feys, L., Descamps, B., Vanhove, C., Vral, A., Veldeman, L., Vermeulen,S., et al. (2015) Radiation-induced lung damage promotes breast cancer lung-metastasisthroughCXCR4 signaling. Oncotarget 6, 26615-26632. doi:10.18632/ oncotarget.5666.

7. Fu Z, Zhen W, Yuskavage J, Liu D. (2010) Epigallocatechin gallate delays the onset of type 1 diabetes in spontaneous non-obese diabetic mice. Br J Nutr; 105:1218-25.

8. Giannelli G, Fransvea E, Marinosci F, Bergamini C, Colucci S, Schiraldi O, et al.( 2002) Transforming growth factor-beta 1 triggers hepatocellular carcinoma invasiveness via alpha3beta1 integrin. Am J Pathol; 161:183-193.

9. Hafezeh Davari, Farhang Haddad, Ali Moghimi et al. (2012) Study of Radioprotective Effect of Green Tea against Gamma Irradiation Using Micronucleus Assay on Binucleated Human Lymphocytes. Iranian Journal of Basic Medical Sciences Vol. 15, No. 5, 1026-1031.

10. Hu, Z.Q., Toda, M., Okubo, S., Hara, Y., Shimamura, T. (2011) Mitogenic activity of (-) epigallocatechin gallate on B-cells and investigation of its structurefunction relationship. Int. J. Immunopharmacol 1992. 14 (8), 1399-1407. 
11. K. Pardali, A. Moustakas. (2007) Actions of TGF-beta as tumor suppressor and prometastatic factor in human cancer, Biochim. Biophys. Acta 1775 1-62.

12. Lal G, Bromberg JS. (2009) Epigenetic mechanisms of regulation of Foxp3 expression. Blood; 114:3727-35.

13. Lambert JD, Yang CS.( 2003) Mechanisms of cancer prevention by tea constituents. J Nutr.; 133:32625-75.

14. Livak K. J., Schmittgen T.D. (2001) Analysis of Relative Gene Expression Data using Real-Time Quantitative PCR and the 2 (-Delta Delta C(T)) Method. Methods (San Diego,Calif.);25(4):402-408.

15. Nakamura K, Kitani A, Fuss I, Pedersen A, Harada N, Nawata H, et al.( 2004) TGF-beta1 plays an important role in the mechanism of CD4CD25 regulatory $\mathrm{T}$ cell activity in both humans and mice. J Immunol; 172: 834-842.

16. Pae M, Ren Z, Meydani M, Shang F, Meydani SN, Wu D.(2010) Epigallocatechin-3-gallate directly suppresses T cell proliferation through impaired IL-2 utilization and cell cycle progression. J Nutr; 140:150915 .

17. Satoshi Kitaia, Masatoshi Kudoa, Naoshi Nishidaa et al. (2016) Survival Benefit of Locoregional Treatment for Hepatocellular Carcinoma with Advanced Liver Cirrhosis, Liver Cancer;5:175-189.

18. Sundaresan S, Subramanian P.( 2003) S-Allylcysteine inhibits circulatory lipid peroxidation and promotes antioxidants in N-nitrosodiethylamineinduced carcinogenesis. Pol J Pharmacol, 55:37-42.

19. Surace, L., Guckenberger, M., and vandenBroek, M. (2015) Radiation holidays stimulate tumor immunity. Oncotarget 6, 15716-15717.doi: 10.18632/oncotarget.4608.

20. Takahashi H., Oyamada M., Fujimoto Y., Satoh M.I., Hattori A., Dempo K., Mori M., Tanaka T., Watabe H., Masuda R., Yoshida M.C.( 1988) Elevation of serum alpha-fetoprotein and proliferation of oval cells in the livers of LEC rats. Japanese journal of cancer research; 79(7): 821-827.

21. Vanessa Crespy, Gray Williamson.( 2004 ) A Review of the health effects of green tea catechins in vivo animal models. J.Nutr.134:3431S-3440S.

22. Wenjun Guo and Filippo G. Giancotti.( 2004) integrin signaling during tumor progression. Cell Biology Program, October, doi:10.1038/nrm1490.

23. World Health Organization (2014). World Cancer Report 2014. Pp. Chapter 1.1.

24. Xiao J, Lu R, Shen $X$ and Wu M. (2002) Green tea extracts protected against carbon tetrachloride-induced chronic liver damage and cirrhosis. Zhonghua Yu Fang Yi Xue Za Zhi 36(4): 243-246. 